\title{
HANDLING HUMAN FACTORS IN INTEGRATED SYSTEMS ENGINEERING
}

Coping with Context-Adaptive Behavior

\author{
Michael Cebulla \\ Technische Universität Berlin, Fakultät IV, Institut für Softwaretechnik und theoretische \\ Informatik, mce@cs.tu-berlin.de.
}

\begin{abstract}
In this paper we focus on architectural concepts for complex sociotechnical systems and advanced pervasive applications which have to be highly context aware. First we claim that there is a great need for model-based reasoning about systemic properties concerning questions of system design and the definition of long-term management policies. After this we take our starting point from formal methods, requirements engineering, and software architecture. We provide special extensions for these methods which are wellsuited for the special challenges of sociotechnical systems: adaptive behavior and the behavioral relevance of cognitive parameters. We maintain the visual style of modeling concepts as known from software architecture and provide elements of an easy to use notation for reasoning about the features of specific situations. Finally we provide concepts to deal with uncertain system behavior and human error.
\end{abstract}

Key words: Methodologies, Context Models, Human Error.

\section{INTRODUCTION}

Modern system engineering is increasingly confronted with a new quality of contextual embedding. For the specification of systemic behavior a growing number of environmental parameters have to be taken into account. We conceive these parameters as systemic aspects and use ontologies to achieve a modular way to manage the related knowledge. In this paper we focus on human factors as a major contextual parameter.

Sociotechnical systems as well as advanced applications like pervasive services have to reside in complex contexts and frequently have to deal with 
unforeseen situations which may lead to error situations. Hence their behavior is deeply interwoven with numerous parameters determined by the external environment. One important special case of contextual dependency is represented by the increased significance of human machine interaction. Advanced pervasive applications with multimodal interfaces will have to be able to maintain complex hypotheses about users and their situation in order to be of any practical value. This new type of application has to provide a behavior which is far more flexible than traditional computer systems. Apart from being context aware in some general sense they have to be aware of the users identity, their capabilities, their goals, and their plans.

Consequently, from the perspective of integrated systems engineering context dependent human machine interaction has to be conceived as a systemic aspect which has gained increased relevance. In addition, in the light of a more intensive aspectual interweaving concepts for human error modeling have to be compatible with concepts for the description of other aspects. On the basis of this compatibility complex interactions (Leveson, 1995; Perrow, 1984) between different types of systemic agents can be analyzed. These interactions are a prominent cause of system failures and losses.

In this paper we focus on the integration of two important aspects of system modeling. By providing ontological concepts for the aspects of workflow and human factors we gain the advantages of modularity, compositionality, and conceptual reusability. On this platform we can integrate concepts related to the description of cognitive states into workflow description.

We claim that our conceptual frameset allows for an integrated view on system behavior with specific consideration of human factors. Since our concepts are abstract and located on the level of conceptual modeling they are equally well suited for the description of several types of hybrid systems where teams of humans cooperate with ensembles of devices. In addition, we claim that our approach based on conceptual modeling is well-suited to specify insights about interactive behavior in sociotechnical systems and transfer them to the design of context aware systems in general.

\section{CONCEPTUAL MODELING}

Originally, we developed our concepts for the analysis and modeling of complex sociotechnical systems. Due to the complexity of these systems their analysis and understanding is very difficult. But on the other hand for various reasons (safety, efficiency, organizational learning, change management) there is a strong need for a greater transparency of the related 
processes. This is shown vividly by the great number of disasters, losses and catastrophes during the recent decades.

In our approach we develop a visual notation for the modeling of complex sociotechnical systems. Starting from formal methods and the experiences of requirements engineering (Partsch, 1998; Pepper, Wirsing, 1995) we provide concepts which are well suited for the description of specific sociotechnical features. Especially the aspects of variability and adaptation but also those of human cognition and organizational relations are traditionally hard to grasp by formal notations. In addition, uncertain system behavior and human error are topics of great relevance.

We claim that a visual modeling notation significantly increases system transparency, support interdisciplinary system analysis, and is well-suited to support measurements of further education. On the long run we plan to provide automated tools for analysis, simulation and the support of system management. For the semantic foundation of our notation (which is beyond the scope of this paper) we use description logic (Baader et al., 2003).

In addition, we claim that our approach of conceptual modeling provides a platform for a better understanding of the impact of human factors on safety-critical features of complex systems in general. The resulting conceptual models of social interaction, organizational dependencies, and human error are equally well-suited as foundations for the design of pervasive and adaptive systems. Since these advanced services have to adapt smoothly to changing contexts they have to possess similar adaptive capabilities as traditional sociotechnical systems. We think that conceptual models of adaptive and context-aware behaviors are well-suited as transfer media for these capabilities. Generally, these conceptual models are major contributions for a better understanding of complex systems and an enhancement of their safety-related properties.

We describe the motivation for our work in sections 2 and 3. In section 4 we identify some specific sociotechnical challenges for model-based methods. While we explain the foundations of our method in sections 5 and 6 , we apply our concepts on specific sociotechnical features in sections 7 to 9.

\section{COMPLEXITY AND SAFETY}

Complex sociotechnical systems have evolved to control high risk technologies by teams of highly qualified specialists. Sociotechnical systems can be defined as complex safety-critical systems where teams of human operators cooperate with ensembles of technical units and devices. Usually, the resulting processes are significantly more complex than in traditional 
systems consisting solely of technological components. Generally, their behavior is more dependent on contextual changes. Thus, a model for these kinds of systems has to take into account not only technology but also contextual parameters as in our case human factors. Examples for this kind of systems are not only atomic power plants, medical operation theaters and air traffic control, but also safety-critical pervasive applications, which we expect to evolve in the near future.

This new classes of systemic complexity, contextual determination and its related risks have established new requirements for system design and system safety. This is documented by the sad history of catastrophes from Three Miles Island (1979) to Überlingen (2002). The analysis of such complex systems has proven too multi-faceted for the traditional singledisciplinary approach.

A model-based interdisciplinary system analysis is a promising strategy against what Leveson calls intellectual unmanageability of high risk systems (Leveson, 1995). The increasing complexity and tight coupling in contemporary high risk systems make a safe and efficient management difficult if not impossible. The main source of failure in complex systems is not human error or an erroneous component, but the complex interactions between components which is not understood to a sufficient degree. To increase the level of understanding we choose a model-based approach which is open for results of interdisciplinary research (i.e. from disciplines like semiotics, psychology or sociology).

\section{CHALLENGES OF SOCIOTECHNICAL SYSTEMS}

Of course, the methods of system modeling are not new. Especially in software engineering concepts and methods were synthesized to handle the challenges of complexity in development processes. Consequently, in our work we heavily rely on the results of system architecture, formal specification and systems engineering (Sage, 1999). The concepts and methods from requirements engineering provide the basic means to manage informal and semiformal knowledge about the target system.

We observed some specific features of sociotechnical system which can be conceived as challenges for traditional modeling concepts. We claim that the traditional modeling concepts and formal methods as known from software engineering have to be adapted and extended for the specific properties of sociotechnical systems. Speaking generally, just the merits of concepts for formal specification as exactness and well-definedness sometimes prove as shortcomings in the context of sociotechnical systems. It 
is a generic feature of these systems that they have to deal with vague data, uncertainty and incomplete specifications. Modeling concepts have to adapt to this specific vagueness which can be conceived as important system quality. Paradoxically speaking, too much exactness would lead to less adequate or even wrong specifications.

\subsection{Uncertainty}

Sociotechnical systems tend to reduce the load of information processing by using vague concepts. So human experts normally don't use exact mathematical expressions (like for example partial differential equations) but vague expressions from natural languages. We claim that the resulting vagueness is an important precondition for the systems robustness and safety since vague specifications are compatible with changing contexts.

In addition, human actors frequently have to deal with incomplete specifications. In many situation relevant information is not accessible for them. Due to situational time pressure they have to make uncertain decisions based on incomplete information (Cebulla, 2003). In our approach we use fuzzy sets and fuzzy logic to specify uncertain information and vague relations (Klir, 1995).

\subsection{Adaptive Behavior}

One important feature of sociotechnical systems is their structural dynamism. The internal structure of systems like the medical operation theatre can be rapidly changing from one phase of the process to another according to environmental changes. For the description of this structural dynamism powerful concepts from dynamic architectures (Pepper, 2003) are necessary. We handle this problem by introducing transformation rules.

By specifying transformation rules we define the possibilities of configurations to evolve under changing context conditions. Hence transformation rules describe the adaptive behavior of complex sociotechnical systems in specifying the way these systems react to environmental changes by structural mutation (for an example cf. section 7).

Sociotechnical systems like the medical operation theatre have distinctive qualities regarding their adaptive capabilities. Thus, their ability to recover in the face of adverse environmental conditions or unexpected events is clearly larger compared to the behavior of traditional component-based systems. Regarding this adaptivity a deeper understanding of sociotechnical processes can contribute to the robustness and flexibility of software-based systems. 


\subsection{Subjective Evidence}

Domain experts tend to disagree about the facts in their field. Sometimes they are not completely sure. We use fuzzy concepts for the representation of different degrees of subjective evidence and relevance. This enables us to distinguish for example between different degrees of adaptation to a given context (cf. Section 6).

\section{BASICS OF ARCHITECTURAL DESCRIPTION}

For the description of a system's structure we use the concepts known from software architecture (Shaw 1996, Pepper 2002). Doing this we apply a well-known method to an uncommon area. By this transfer we reuse the competence that was gained on the field of software engineering for the structural description of sociotechnical systems. By adopting these concepts we establish an ontology for the conceptual modeling of complex systems.

We conceive the structure of a system as a configuration of agents which are interacting using connectors. By providing different types of agents and connectors with different signatures (or interfaces) we are able to define semantically meaningful types of configurations (usually called architectural styles) by composition. Both, agents and connectors publish interfaces (specified by algebraic signatures) which may be connected by links (cf Figure 1).

First we provide a basic vocabulary of agent and connector types which we found suitable for the description of a given domain (cf. Figure 3 for an example). We notate agents as soft-boxes and connectors as ovals. Interfaces are represented by little squares.

As an example we choose the setting of a medical operation theatre. While this special system is characterized by a great variability we have to content ourselves with describing exemplary scenarios taken from anesthesia.

We describe interfaces using a tabular specification: each variable and action name is listed with its name and its type (cf. Figure 2). Thus, for example the anesthesist can use his voice to give some commands (give, take) or use his visual sense to observe the color of the patient's face. In the given situation he is also able to perform some actions (intubating the patient or configure the monitoring device).

Every interface is described by a set of input and output variables and a set of action names which specify his capabilities to interact with his environment by physical channels. This visual specification technique is fully compatible with the more formal methods of algebraic specification. 
We use features (also called port variables) to describe the physical qualities and services of agents. The left hand of an actor or the displays of a technical device are examples for this kind of variable. The domain of these variables frequently is defined by enumeration types which may have fuzzy semantics (Klir, 1995).

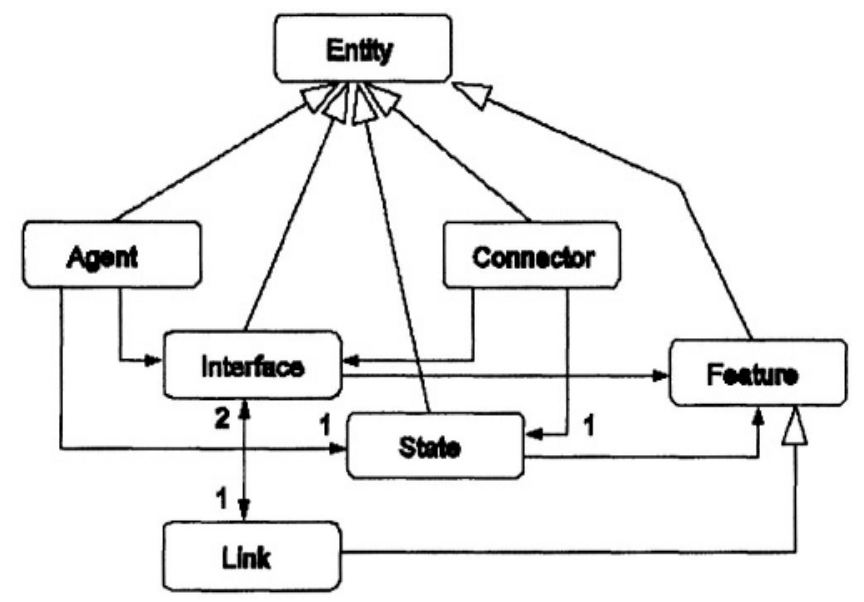

Figure 1. Metamodel for Systems Analysis

As we show in Figure 1 we conceive an ontology as a collection of entities. Each of the syntactic elements presented in this section is an extension of entity. In addition we make some statements about the way these concepts are combined. Agents as well as connectors may possess a set of interfaces, which in turn contain a set of features. These aggregation relations possess the cardinality $0 \ldots n$ which we conceive as default and which is ignored in the figure. Agents and Connectors may contain a state which in turn contains a set of features. Links as special cases of features connect exactly two interfaces.

We conceive this framework as a starting point for the definition of domain-specific ontologies which consists of typed specializations of these abstract concepts (i.e. HumanActor, Anesthesist, or Communication). 


\begin{tabular}{|c|c|}
\hline \multicolumn{2}{|c|}{ Interface IA1 } \\
\hline \multirow{4}{*}{$\frac{3}{5}$} & vis:\{pale, red, normal\} \\
\hline & acc:\{lowPitch, highPitch\} \\
\hline & tact: $\{$ wet, normal\} \\
\hline & olf:\{normal, narcotics\} \\
\hline \multirow{4}{*}{ 蛋 } & voc: \{glve, take\} \\
\hline & bodytalk:\{calm, nervous $\}$ \\
\hline & Iman: \{fixing, nop\} \\
\hline & rman: \{holdTube, introTube, nop\} \\
\hline \multirow{4}{*}{$\frac{y}{5}$} & voc: $\{$ give, take $\}$ \\
\hline & take: $\{$ rman $\}$ \\
\hline & introTube: $\{$ rman, vis\} \\
\hline & configMonitoring: $\{r m a n$, vis $\}$ \\
\hline
\end{tabular}

Figure 2. Tabular Specification of an Interface

\section{MENTAL MODELS: A COGNITIVE APPROACH}

We provide a cognitive perspective which takes into account an agent's subjective motivations and their influence on the global system's behavior as major contextual parameters. We claim that this allows for a better understanding of the contextual determination of systemic behavior. Especially the human factors and organizational relationships in sociotechnical processes can be modeled by these concepts. For this sake we adopt the concepts related mental models as known from the BDI-style frequently used in agent oriented modeling (Singh, 1994).

An agent's mental model consists of:

- The agent's intentions: usually a decision aims at a certain goal. That means that the agent tries to achieve a certain system state by selecting between alternative behavioral options.

- The agent's beliefs (also: expectations): a decision is highly influenced by the agent's belief concerning the system's actual state and its further behavior.

- The agent's desires: usually the agent has a subjective preference for a certain behavioral option which may or may not interfere with the real situation.

We represent this internal information using fuzzy sets. This gives us the possibility to represent the subjective relevance of a proposition by the membership relation $\mu$ (Sperber, Wilson, 1986). 
We notate fuzzy sets by using bold font. Following a widespread convention we occasionally use the name of the set as a shortcut for its membership function. The function of the three fuzzy sets $\mathbf{B}, \mathbf{D}$ and $\mathbf{I}$ consists in the mental representation of the relevant contextual features.

- Fuzzy set I represents the agent's intentions.

- Fuzzy set B represents the agent's expectations concerning the system's actual state.

- Fuzzy set $\mathbf{D}$ represents the agent's desires (preferences) concerning the behavioral options. These preferences are usually context independent.

In Figure 3 we use these concepts for the definition of an actors's adaptation.

We claim that in order to be well adapted the actors's internal representations have to be very close to the relevant features of the given context. Especially the actor has to have an intensive internal representation of the global system goals (first condition) and has to be well informed regarding the relevant features of a given context (second condition).

Alternatively she may have a strong inclination for the right behavioral option (third condition). In every case the relevant propositions have to be members of the nurse's mental model to a high degree. We say that the nurse is well adapted to her context if the first and second conditions or the third condition are given.

As in Figure 3 we use fuzzy rules to approximate the relation between contextual features and an actor's mental representations. In general, we claim that an actor's contextual adaptation is good if he has in intensive representation of the relevant features. We use AND/OR-tables to reason about different configurations and their consequences for the quality of adaptation. There we use the letters $\mathrm{T}$ and $\mathrm{F}$ to mark the conditions which are true resp. false. Conditions that are not relevant in a given configuration we mark with a star. To qualify key features of a configuration we use the symbolic constants high $(h)$, medium $(m)$ and low $(l)$.

For organizational purposes there are some interesting features of contexts. The cardinality of set $A$ describes the free room of an agent's decision. If it is small this has inhibiting effects on his motivation. If it is too great the agent may be overstrained. Moreover, another important feature of a given context consists in the possibility of differentiation of behavioral alternatives. 


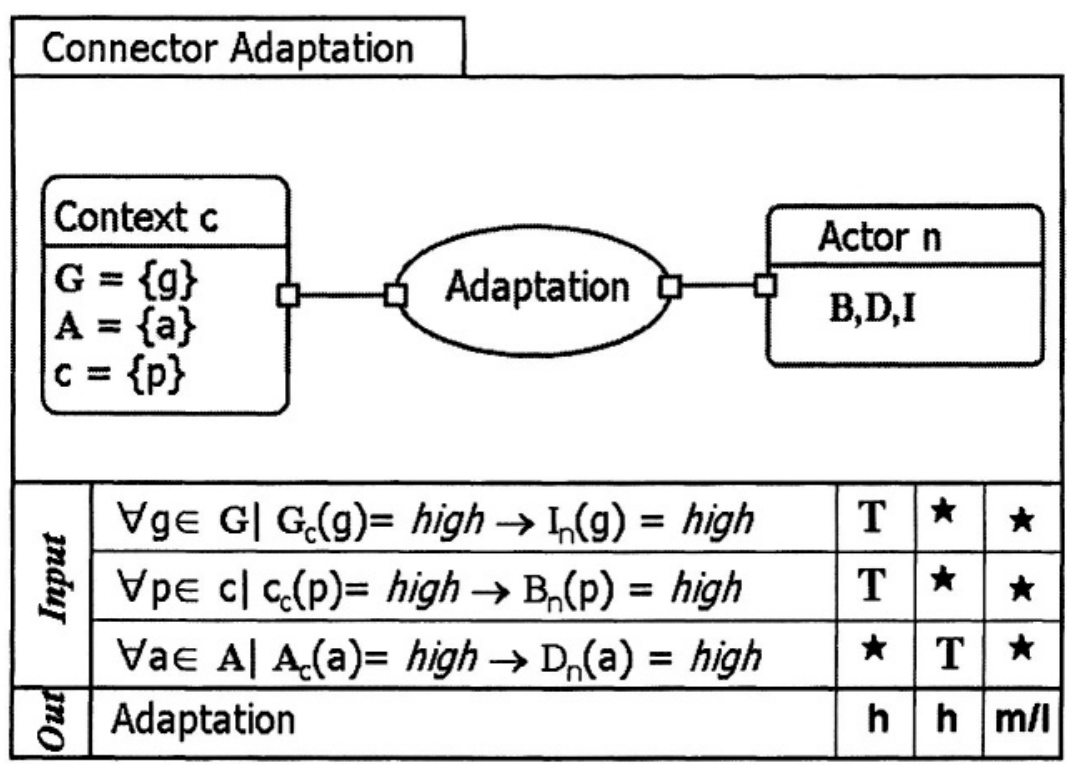

Figure 3. Adaptation

\section{CONTEXTUAL DEPENDENCIES}

For the processing of systemic tasks agents and devices constitute configurations by establishing interaction networks using connectors. In order to model the adaptive capabilities of sociotechnical systems as well as the contextual dependencies of configurations we distinguish between abstract models and situational models (adopting analogous concepts from Soley, 2000). We provide concepts for the definition of context-specific transformation rules which transfer abstract specification into situational descriptions according to contextual conditions.

Hence, in Figure 4 we show a transformation rule which defines the adaptive capabilities of a given configuration with respect to certain adversal context conditions. In our example, at the beginning of a given task a necessary precondition is not given. The laryngoscope (a special instrument which is used during intubation) is not owned by the anesthesist. 


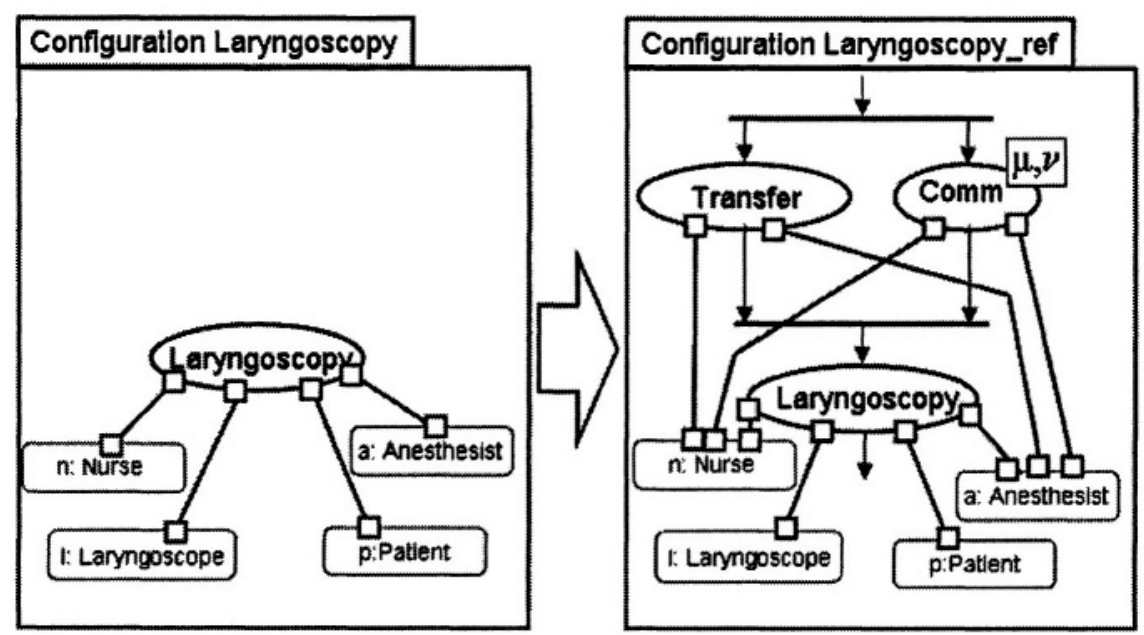

precondition: l.owner $=$ nurse AND $\mathbf{I}_{n}(1$. owner=a) $=$ NULL

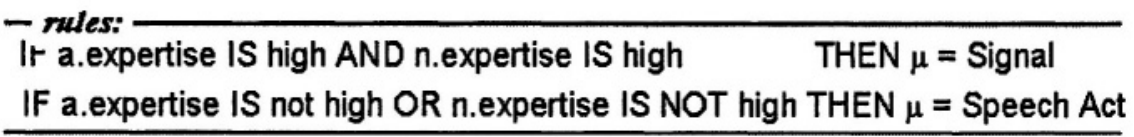

Figure 4. Transformation Rule for Adaptive Behavior

In order to compensate this adversal condition we have to change the task structure by the application of our transformation rule. We generate two coordinative task which establish the desired state of affairs. The way in which this is done highly depends on the capabilities and internal representations of the agents involved. Thus we have to generate a communicative task because in our example the nurse isn't aware of the necessity to give the laryngoscope to the anesthesist (as stated in the precondition).

Intuitively, in Figure 4 we describe the case that the nurse possesses the device in the wrong moment and is not aware of necessity to give it to the anesthesist. We claim that this situation is characterized by strong adverse conditions which the system has to compensate by context-adaptive behavior. We describe one possible behavioral alternative in our rule.

Using this kind of rule-based specification allows us to catch the contextsensitivity of task processing. Which type of procedure is used by the actors in a specific situation is highly dependent on the parameters of the context. One example for such parameters is the expertise of the actors: highly trained experts tend to coordinate their activities using signals, while novices normally use convention-based speech acts. This rule is very similar to configuration rules in feature modeling (Czarnecki, Eisenecker 2000). 


\section{SOCIOTECHNICAL CONNECTORS: COMMUNICATION}

We conceive connectors as specifications about the behavior of agents. We use temporal logics to define the required behavioral properties. In our example (Figure 4) we use enabling relations inspired by event structures (Winskel, 1988) which allows us to specify partial orders over events.

An important relation between agents is Communication. For the definition of this relationship we have to use our operators from epistemic logic to specify formulas about the mental models of agents.

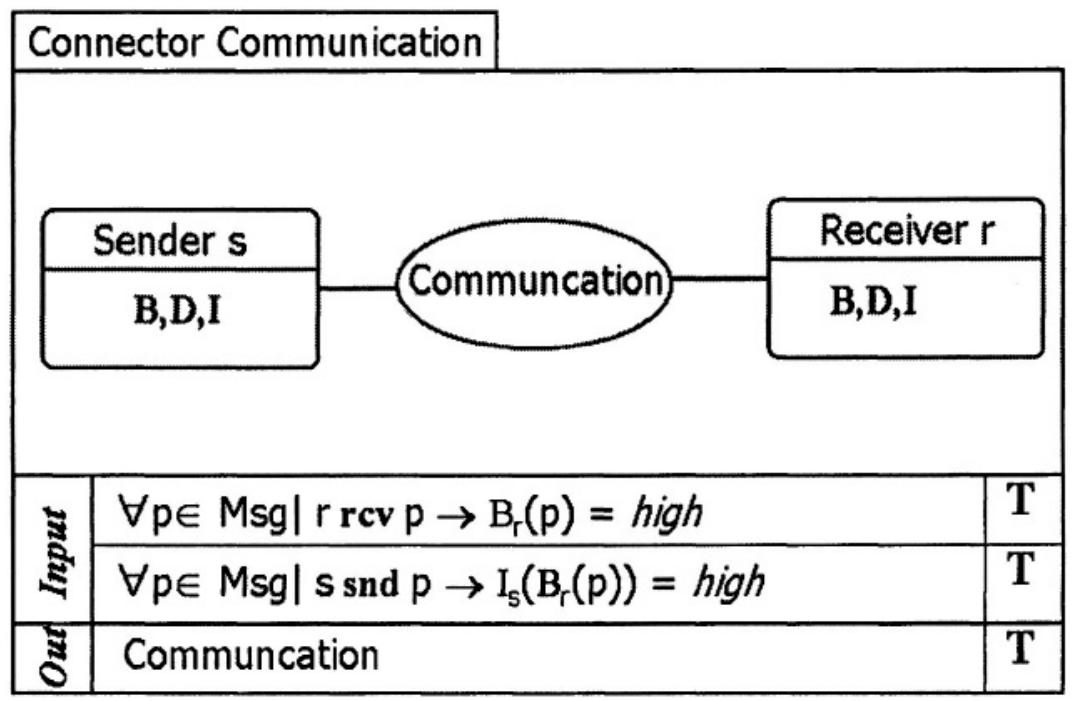

Figure 5. Connector Communication

Our first input condition in Figure 5 specifies what is known as the perlocution condition from speech act theory: a result of a communication process is that the message's recipient believes in the content of the message because he received this message. Using our terminology we claim that the recipient of message beliefs its content when the proposition is member in his set of beliefs $\mathbf{B}$ to a high degree.

The second condition is called illocution condition: for a communication act it is a necessary condition that it belongs to the actor's goals to induce the receiver's conviction that the proposition $p$ holds.

This formalization of communication processes is an exemplary result of interdisciplinary cooperation. We introduced semiotic concepts in our modeling framework which are presented in (Cebulla, 1995). 


\section{UNCERTAIN BEHAVIOR AND HUMAN ERROR}

Unlike deterministic behavior of technical devices human decision making is a source of behavioral uncertainty. The outcome of an actor's decision is highly unpredictable and depends on numerous parameters which are not directly observable (as for example mental models).

Since we have a special interest in the safety analysis of sociotechnical systems we use our modeling framework for the analysis of human error. We reason about errors using fuzzy fault trees. Using fault trees we can reason about different types of human errors using propositions about mental models. As shown in Figure 9 we distinguish between three cases of human error which are grouped in two sections following (Reason, 1990):

- Execution-based errors (slips) occur when the subjective preferences of an actor are not adapted to the context (goals and beliefs may be fitting).

- Knowledge-based errors (mistakes) may occur when the beliefs of an actor are not adapted to the given context. For example a nurse may have the right goals but may not act right because she doesn't see the need to act. Another error-case occurs when an actor's goals do not fit well into the given context.

We claim that the integration of cognitive parameters like mental models into our modeling formalism provides a foundation for the reformulation of interdisciplinary results from semiotics, sociology or psychology. Note that we want to provide a conceptual framework which allows us to consider these aspects into the integrated modeling of complex systems. As a specific benefit of this integration we conceive the modeling of complex interactions as for example the situational interplay of human factors and physical properties of technical devices. A modeling framework which allows for this integration is a necessary precondition for the systematic analysis of this systemic couplings and side-effects.

Thus, we suggest that the use of integrated fuzzy fault trees is an essential method for an interdisciplinary scenario based system analysis. We claim that the impact of misplaced cognitive representations on the overall systemic behavior can be analyzed using these concepts. 


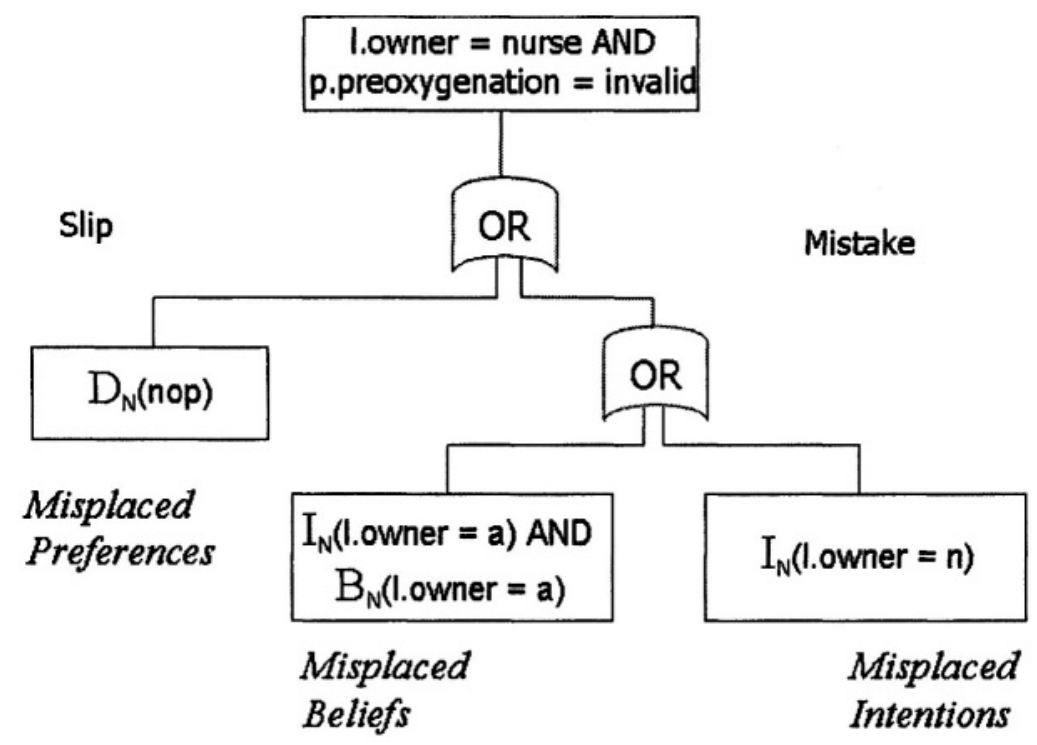

Figure 6. Fuzzy Fault Tree for Human Error

\section{CONCLUSION}

In this paper we provided and demonstrated elements of a visual notation for the integrated analysis of complex sociotechnical systems as well as for advanced context aware applications. We started with structural description, dynamic architectures, and rules for reasoning about adaptive system properties. For this sake we extended common architectural concepts by fuzzy methods and a transformational approach. We then took a cognitive perspective by introducing the concepts of mental models. At this point we used results from psychology, sociology and semiotics.

On this platform we provided modeling concepts for specific human and organizational relationships. We conceived for example communication as coordination mechanism which makes it possible for agents to balance their intentions and beliefs concerning the further processing of the system's task.

Using this model we can reason about the system's safety-critical features. As we demonstrated each mechanism acts as an context adaptive mechanism in the face of adverse environmental condititons concerning the system's safety. We also call these mechanisms safety barriers. Finally, we sketched our way to deal with uncertain behavior and human error. 


\section{ACKNOWLEDGEMENTS}

This work was funded by Technical University of Berlin in the context of an interdisciplinary research focus Cooperation and Safety for Complex Sociotechnical Systems (KOSIS). I am very grateful for the cooperation with my colleagues from Ergonomics, Semiotics, Sociology and Psychology. I am also indebted to the anonymous reviewers for their constructive criticism.

\section{REFERENCES}

Baader, F., Calvanese, D., McGuiness, D., Nardi, D., Patel-Schneider, P. The Description Logic Handbook. Theory, Implementation and Applications. Cambridge Univ. Pr. 2003.

Cebulla, M. "Pragmatik und KI: Posners semiotische Fundierung der Sprechakttheorie." $S$ European Journal for Semiotic Studies. Vol. 7, No. 1,2,1995.

Cebulla, M. "Reasoning about Variability and Structural Adaptation in Sociotechnical Systems using Dynamic Architectures.” Proc. of SEKE'03, Redwood City, Ca. 2003.

Czarnecki, K., Eisenecker, U. Generative Programming. Methods, Tools, and Applications. Addison Wesley: Reading, Mass., 2000.

Klir, G., Yuan, B., Fuzzy Sets and Fuzzy Logic. Theory and Applications. Prentice Hall: Upper Saddle River, N.J. 1995.

Leveson, N., Safeware. System safety and computers. Addison Wesley: Reading, Mass. 1995.

Leveson, N., A new Accident Model for Engineering Safer Systems. In preparation 2002.

Minzberg, H. Structures in fives: designing effective organizations. Prentice Hall: Englewood Cliffs, 1983

Partsch, H., Requirements Engineering systematisch. Springer, Berlin u.a., 1998.

Pepper, P., Wirsing, M, "A method for the development of correct software", In: Manfred

Broy, Stefan Jähnichen (eds.), KORSO: Methods, Languages, and Tools for the

Construction of Correct Software. Springer: Berlin u.a., 1995

Pepper, P., Cebulla, M., Didrich, K., Grieskamp, W. "From Program Languages to Software Languages", The Journal of Systems and Software, 60,2002.

Pepper, P, Frank, C., Holfelder, W., Jiang, D., Matylis, G. Dynamic Architectures for a „,sometimes somewhere “ concept. Technical Report, TU Berlin, 2003.

Perrow, C. Normal Accidents. Living with High Risk Technologies. Basic Books: New York, 1984.

Reason, J. Human Error. Cambridge Univ. Press, 1990.

Sage, A.P., Rouse, W.B. (eds.) Handbook of Systems Engineering and Management. Wiley: New York. 1999.

Shaw, M, Garlan, D. Software Architecture. Perspectives on an emerging discipline. Prentice Hall: Upper Saddle River, N.J., 1996.

Singh, M.P., Multiagent Systems. A Theoretical Framework for Intentions, Know-how, and Communications. Springer: Berlin, 1994.

Soley, R. "Model Driven Architecture, White Paper." ftp://ftp.omg.org/pub/docs/omg/00-1105.pdf (23.3.2004).

Sperber, D., Wilson, D. Relevance. Communication and Cognition. Harvard Univ. Pr.: Cambridge, Mass, Blackwell: Oxford, 1986. 
Winskel, G. "An Introduction to Event Structures", in: Linear Time, Branching Time, and Partial Order in Logics and Models for Concurrenc., Springer: Berlin, 1988. 